\title{
A framework radiation hybrid map of buffalo chromosome 1 ordering scaffolds from buffalo genome sequence assembly
}

N.B. Stafuzza' ${ }^{1}$ B.C.M. Naressi ${ }^{1}$, E. Yang ${ }^{2}$, J.J. Cai ${ }^{2}$ and M.E.J. Amaral-Trusty ${ }^{1}$

1Departamento de Biologia, Instituto de Biociências, Letras e Ciências Exatas, Universidade Estadual Paulista Júlio de Mesquita Filho,

São José do Rio Preto, SP, Brasil

${ }^{2}$ Department of Veterinary Integrative Biosciences, Texas A\&M University,

College Station, TX, USA

Corresponding author: M.E.J. Amaral-Trusty

E-mail: eamaral@ibilce.unesp.br

Genet. Mol. Res. 14 (4): 13096-13104 (2015)

Received April 20, 2015

Accepted August 12, 2015

Published October 26, 2015

DOI http://dx.doi.org/10.4238/2015.October.26.5

ABSTRACT. River buffalo chromosome 1 (BBU1) is a sub-metacentric chromosome homologous to bovine chromosomes 1 and 27 . In this study, we constructed a new framework radiation hybrid $(\mathrm{RH})$ map from BBU1 using BBURH ${ }_{5000}$ panel adding nine new genes (ADRB3, ATP2C1, COPB2, CRYGS, P2RY1, SLC5A3, SLC20A2, SST, and ZDHHC2) and one microsatellite (CSSM043) to the set of markers previously mapped on BBU1. The new framework RH map of BBU1 contained 141 markers (55 genes, 2 ESTs, 10 microsatellites, and 74 SNPs) distributed within one linkage group spanning 2832.62 centirays. Comparison of the $\mathrm{RH}$ map 
to sequences from bovine chromosomes 1 and 27 revealed an inversion close to the telomeric region. In addition, we ordered a set of 34 scaffolds from the buffalo genome assembly UMD_CASPUR_WB_2.0. The RH map could provide a valuable tool to order scaffolds from the buffalo genome sequence, contributing to its annotation.

Key words: Bubalus bubalis; Chromosome 1; Comparative map; Radiation hybrid mapping

\section{INTRODUCTION}

Buffalo (Bubalus bubalis) plays an important role in world economy, providing highquality milk and meat, mainly in countries with less financial resources for livestock research. Its genome is organized in five pairs of bi-armed chromosomes, 19 pairs of acrocentric chromosomes, and a pair of $X$ and $Y$ sex chromosomes. The largest chromosome in the river buffalo karyotype, BBU1, is a sub-metacentric chromosome with reported homology between BBU1q and bovine chromosome 1 and between BBU1p and bovine chromosome 27 (lannuzzi et al., 2003; Miziara et al., 2007).

Genome analysis of $B$. bubalis has advanced significantly in the last few years with the availability of genomic tools such as a radiation hybrid $(\mathrm{RH})$ panel for whole genome mapping (Amaral et al., 2008; Stafuzza et al., 2009) and a genomic BAC library (Stafuzza et al., 2012) especially useful for region-specific re-sequencing and characterization of target genes (Stafuzza et al., 2014a,b,c).

In 2014, a buffalo whole genome sequence generated by next-generation sequencing platforms was publicly released in the NCBI database (http://www.ncbi.nlm.nih.gov/ assembly/67671). Nevertheless, mapping of the buffalo genome remains important for identification of genes associated with complex economic traits and for evaluating chromosomal evolution among species of the Bovidae family. Considering that buffalo and cattle exhibit an evolutionary divergence estimated at 20 million years (Parma et al., 2004), investigations on chromosomal rearrangements and comparison of position and order of homologous genes are valuable to enhance our understanding of both the genomes.

In the present study, we constructed a new framework $\mathrm{RH}$ map from BBU1 using $\mathrm{BBURH}_{5000}$ panel (Amaral et al., 2007), mapping ten new markers including nine coding genes and one microsatellite. The RH map was compared to the sequence from bovine chromosomes 1 and 27 and used to order scaffolds from the buffalo genome assembly UMD_CASPUR_WB_2.0 available in the NCBI database.

\section{MATERIAL AND METHODS}

Nine new genes (ADRB3, ATP2C1, COPB2, CRYGS, P2RY1, SLC5A3, SLC20A2, SST, and $Z D H H C 2)$ and one microsatellite (CSSM043) derived from bovine chromosomes 1 and 27 
were typed by polymerase chain reaction (PCR) on the buffalo RH panel (Amaral et al., 2007). The reference for each marker is listed in Table 1.

PCR was performed in a Veriti ${ }^{\circledR}$ Thermal Cycler (Life Technologies ${ }^{\mathrm{TM}}$, Carlsbad, CA,USA) with thermal gradient software. Bovine DNA samples were included as controls in each amplification experiment since the PCR primers were cattle-derived. The markers were scored after amplification of DNA from $90 \mathrm{RH}$ cell lines and buffalo and hamster control DNA. The PCR mixtures included: $10 \mathrm{mM}$ Tris- $\mathrm{HCl}, 1.5 \mathrm{mM} \mathrm{MgCl}, 50 \mathrm{mM} \mathrm{KCl}, 10 \mathrm{mM}$ dNTPs, 0.2 mM each primer, 0.5 unit AmpliTaq Gold ${ }^{\circledR}$ DNA polymerase (Life Technologies ${ }^{\top \mathrm{M}}$ ), and $50 \mathrm{ng}$ DNA in a $10 \mu \mathrm{L}$ reaction volume. The PCR conditions were as follows: initial denaturation at $94^{\circ} \mathrm{C}$ for $10 \mathrm{~min}$, followed by 35 cycles at $94^{\circ} \mathrm{C}$ for $30 \mathrm{~s}$ (denaturation), $50-65^{\circ} \mathrm{C}$ for $30 \mathrm{~s}$ (annealing depending on the individual marker as described in Table 1), extension at $72^{\circ} \mathrm{C}$ for $30 \mathrm{~s}$, and a final extension at $72^{\circ} \mathrm{C}$ for $7 \mathrm{~min}$. The PCR products were electrophoresed on $2 \%$ agarose gels in $1 \mathrm{X}$ TBE buffer containing ethidium bromide and photographed under UV light. PCR products were scored as 0 for absent, 1 for present, and 2 for ambiguous amplification. All primer sets were typed twice with the RH panel DNA and scored independently to increase the accuracy of the results. Primer pairs that showed ambiguous results were typed a third time.

The RH map was constructed using the software rh_tsp_map (Schäffer et al., 2007) and CONCORDE (Applegate et al., 1998) linked to Qsopt (http://www.tsp.gatech.edu/concorde.html), adding the 10 new markers to the set of markers previously mapped on BBU1 (Amaral et al., 2008). We used the maximum likelihood estimation (MLE) criterion for creating framework MLEconsensus map (Agarwala et al., 2000). Considering the number of markers, the linkage group was made using a pairwise LOD score (logarithm of the odds) threshold of 8.0. The maps were drawn using the MapChart version 2.1 software (Voorrips, 2002).

The BBU1 RH map was compared to the sequence from bovine chromosomes 1 and 27 and used to order scaffolds from the buffalo genome assembly (UMD_CASPUR_ WB_2.0).

\section{RESULTS}

The framework RH map of BBU1 constructed in this study contained 141 markers (55 genes, 2 ESTs, 10 microsatellites, and 74 SNPs) distributed within one linkage group spanning 2832.62 centirays (cR; Figure 1). The retention frequency of the mapped markers ranged from $20.0 \%$ for $M X 1$ and SEC62 to $51.1 \%$ for IFNGR2 gene. Additional information about all the mapped markers, including their retention frequency and $\mathrm{CR}$ position on the map, is compiled in Table 1.

To compare the BBU1 RH map to sequences from bovine chromosomes 1 and 27 (Btau_4.6.1), we constructed a map in mega bases (Mb) using the corresponding positions of the markers available in the NCBI database (Table 2).

A total of 34 scaffolds from the buffalo genome assembly (UMD_CASPUR_WB_2.0) were ordered on BBU1 following the order of the markers on the $\mathrm{RH}$ map (Table 3). 


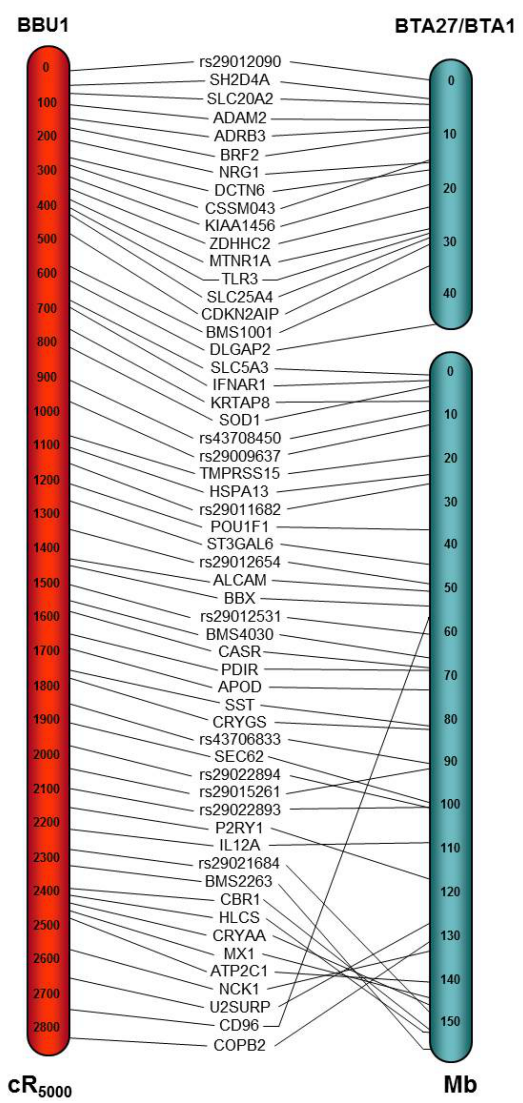

Figure 1. Comparison of BBU1 framework RH map (left) with the cattle chromosomes 1 and 27 from genome build Btau_4.6.1 (right). Positions in the RH map are in cR5000, while positions in the BTA maps are in megabases (Mb). For better illustration, the BBU RH map shows one marker per 50cR. Markers common on both BBU RH and cattle sequence maps are joined by a solid black line.

Table 1. New cattle-derived markers mapped on BBU1 with the BBURH5000 panel, along with their retention frequencies (RF \%), PCR primer references and PCR annealing temperatures (Tm).

\begin{tabular}{|c|c|c|c|c|}
\hline \multicolumn{2}{|r|}{ Marker } & \multirow[t]{2}{*}{ PCR primers reference } & \multirow[t]{2}{*}{ RF (\%) } & \multirow[t]{2}{*}{$\operatorname{Tm}\left({ }^{\circ} \mathrm{C}\right)$} \\
\hline Symbol & Name & & & \\
\hline ADRB3 & Adrenergic, beta-3, receptor & Everts-van der Wind et al., 2004 & 34.4 & 54 \\
\hline ATP2C1 & ATPase, $\mathrm{Ca}++$ transporting, type $2 \mathrm{C}$, member 1 & Ma et al., 1998 & 23.3 & 56 \\
\hline CSSM043 & Microsatellite & Barendse et al., 1994 & 32.2 & 58 \\
\hline COPB2 & Coatomer protein complex, subunit beta 2 & Everts-van der Wind et al., 2004 & 41.1 & 56 \\
\hline CRYGS & Crystallin, gamma S & $\begin{array}{l}{ }^{1} \mathrm{~F}: \text { AGTATCCTGAGTACCAGCACTG } \\
\text { R: TGACTGAAGTCTCAGCAGCCAA }\end{array}$ & 25.5 & 65 \\
\hline P2RY1 & Purinergic receptor P2Y, G-protein coupled, 1 & $\begin{array}{l}{ }^{1} \mathrm{~F} \text { : CTTGTGAAGATGCAGGAATCCC } \\
\text { R: CACGAGGTGTAGGCATTTCCAC }\end{array}$ & 24.4 & 58 \\
\hline SLC5A3 & Solute carrier family 5 , member 3 & Jann et al., 2006 & 43.3 & 50 \\
\hline SLC20A2 & Solute carrier family 20, member 2 & Everts-van der Wind et al., 2004 & 30.0 & 58 \\
\hline SST & Somatostatin & Stafuzza et al., 2014a & 24.4 & 60 \\
\hline ZDHHC2 & Zinc finger, DHHC-type containing 2 & Everts-van der Wind et al., 2004 & 37.7 & 60 \\
\hline
\end{tabular}

1Designed using Primer3plus (Untergasser et al., 2007), based on the gene sequence from Bos taurus UMD_3.1 reference assembly (GenBank accession No AC_000158.1). 
Table 2. Framework markers mapped on BBU1 and their respective position on BBU1 (cR5000) and on bovine sequence from chromosomes 1 and 27 (Btau_4.6.1).

\begin{tabular}{|c|c|c|}
\hline Marker & Position on BBU1 (cR5000) & Position on Bos taurus genome (bp) \\
\hline BBU1p & & BTA27 \\
\hline rs29012090 & 0.00 & 43633072 \\
\hline rs29025654 & 7.96 & 43629497 \\
\hline rs29020649 & 31.33 & 41093242 \\
\hline SH2D4A & 42.52 & 40495081 \\
\hline THAP1 & 53.53 & 39760978 \\
\hline SLC20A2 & 64.54 & 39466243 \\
\hline rs29013339 & 79.14 & 39532022 \\
\hline ADAM2 & 97.38 & 36663247 \\
\hline ADRB3 & 140.00 & 35215574 \\
\hline CSSM036 & 153.51 & 35129771 \\
\hline BRF2 & 167.14 & 35109839 \\
\hline rs29012498 & 184.44 & 32961800 \\
\hline rs29012293 & 203.18 & 33393165 \\
\hline rs29024459 & 229.71 & 32281344 \\
\hline NRG1 & 241.32 & 29416336 \\
\hline DCTN6 & 255.61 & 28223036 \\
\hline CSSM043 & 266.70 & 29861771 \\
\hline WRN & 283.26 & 29024009 \\
\hline KIAA1456 & 309.04 & 25830826 \\
\hline rs29016185 & 325.99 & 24178540 \\
\hline $\mathrm{ZDHHC2}$ & 342.32 & 21578329 \\
\hline CNOT7 & 355.23 & 21558961 \\
\hline MTNR1A & 374.29 & 17584825 \\
\hline rs29013544 & 385.30 & 17250896 \\
\hline TLR3 & 399.63 & 17342873 \\
\hline SLC25A4 & 416.35 & 16657012 \\
\hline BM1856 & 432.15 & 17420230 \\
\hline ODZ3 & 450.96 & 14505500 \\
\hline CDKN2AIP & 475.07 & 15423000 \\
\hline rs29010442 & 494.33 & 15461470 \\
\hline rs29012623 & 512.83 & 12210114 \\
\hline DEFB1 & 539.80 & 7415644 \\
\hline BMS1001 & 571.60 & 11835596 \\
\hline DLGAP2 & 611.03 & 556266 \\
\hline CLN8 & 620.61 & 1111548 \\
\hline BBU1q & & BTA1 \\
\hline SLC5A3 & 669.05 & 40417 \\
\hline IFNAR1 & 693.38 & 1268604 \\
\hline AW267109 & 714.60 & 1299641 \\
\hline IFNGR2 & 724.07 & 1176873 \\
\hline KRTAP8 & 755.03 & 3828967 \\
\hline rs29013376 & 772.49 & 3548655 \\
\hline TGLA49 & 782.14 & 3267630 \\
\hline SOD1 & 808.70 & 2902719 \\
\hline rs29012842 & 850.24 & 3073723 \\
\hline rs29010139 & 857.88 & 3489251 \\
\hline rs29012844 & 870.64 & 3073460 \\
\hline rs29025993 & 891.77 & 5297323 \\
\hline rs 43708450 & 904.92 & 6306960 \\
\hline rs29013360 & 915.37 & 8023064 \\
\hline rs29013440 & 925.90 & 7923870 \\
\hline AJ496763-027.SP6-429 & 939.15 & Unknown \\
\hline rs29009637 & 968.29 & 11228029 \\
\hline rs29013338 & 1016.33 & 14723290 \\
\hline rs29013314 & 1039.31 & 16827182 \\
\hline rs29019581 & 1050.92 & 16917608 \\
\hline TMPRSS15 & 1068.22 & 18220248 \\
\hline RM095 & 1086.93 & 19538641 \\
\hline HSPA13 & 1102.32 & 22524113 \\
\hline
\end{tabular}

Continued on next page 
Table 2. Continued.

\begin{tabular}{|c|c|c|}
\hline Marker & Position on BBU1 (cR5000) & Position on Bos taurus genome (bp) \\
\hline rs29026806 & 1124.27 & 22002538 \\
\hline rs29011682 & 1147.65 & 24884732 \\
\hline rs43709788 & 1161.97 & 24238388 \\
\hline rs29013247 & 1178.97 & 26816631 \\
\hline rs29012747 & 1201.58 & 35117150 \\
\hline POU1F1 & 1209.49 & 35723143 \\
\hline rs43710099 & 1226.08 & 34312971 \\
\hline PROS1 & 1243.08 & 38728912 \\
\hline ST3GAL6 & 1257.68 & 43994350 \\
\hline rs29013985 & 1266.17 & 44609625 \\
\hline FILIP1L & 1274.25 & 45119183 \\
\hline rs29013984 & 1291.25 & 44609344 \\
\hline rs29010923 & 1319.30 & 47123631 \\
\hline rs29012653 & 1327.80 & 48804954 \\
\hline rs29012654 & 1341.75 & 48805054 \\
\hline rs29017614 & 1400.14 & 52511890 \\
\hline ALCAM & 1430.18 & 50417917 \\
\hline BBX & 1444.02 & 52670743 \\
\hline LOC151584 & 1454.86 & 52966936 \\
\hline rs29013666 & 1463.36 & 54341564 \\
\hline rs29026512 & 1482.24 & 59000296 \\
\hline rs43709748 & 1494.98 & 59799461 \\
\hline rs29012531 & 1504.34 & 59725040 \\
\hline BMS527 & 1516.78 & 62536761 \\
\hline BM1312 & 1534.75 & 61174083 \\
\hline BMS4030 & 1552.05 & 65413568 \\
\hline rs29013617 & 1572.74 & 68201840 \\
\hline CASR & 1581.83 & 67574173 \\
\hline rs43705567 & 1607.14 & 68447239 \\
\hline PDIR & 1648.84 & 68394647 \\
\hline KALRN & 1670.37 & 69451283 \\
\hline rs43710098 & 1678.71 & 71574739 \\
\hline APOD & 1690.32 & 73019925 \\
\hline rs43709791 & 1709.58 & 76197996 \\
\hline rs43708475 & 1725.92 & 78106961 \\
\hline rs29009958 & 1735.39 & 78856792 \\
\hline SST & 1762.02 & 81184020 \\
\hline CRYGS & 1770.67 & 82232306 \\
\hline rs29015647 & 1779.40 & 82593594 \\
\hline rs29022387 & 1792.18 & 88075394 \\
\hline rs 43705570 & 1802.41 & 85828502 \\
\hline rs29009859 & 1835.02 & 89329402 \\
\hline rs43706833 & 1851.31 & 89898926 \\
\hline rs29010311 & 1860.75 & 92071361 \\
\hline rs29019397 & 1873.10 & 93781792 \\
\hline rs29025711 & 1888.63 & 97462173 \\
\hline SEC62 & 1908.87 & 99158891 \\
\hline rs29025486 & 1925.21 & 99613024 \\
\hline rs29020368 & 1933.71 & 101031521 \\
\hline rs29022894 & 1975.25 & 100428314 \\
\hline rs29015261 & 2040.34 & 91411837 \\
\hline rs29022893 & 2099.84 & 100428167 \\
\hline rs29012901 & 2131.51 & 109599237 \\
\hline rs43702504 & 2143.87 & 115024260 \\
\hline P2RY1 & 2156.23 & 116995048 \\
\hline rs42712516 & 2172.12 & 121129184 \\
\hline rs29012024 & 2188.02 & 118649544 \\
\hline IL12A & 2220.19 & 108584853 \\
\hline rs29021684 & 2279.49 & 146683590 \\
\hline C1H8orf42 & 2303.82 & 149961374 \\
\hline BMS2263 & 2327.50 & 156871277 \\
\hline rs29010039 & 2336.93 & 157546306 \\
\hline AF440368-538 & 2381.62 & Unknown \\
\hline CBR1 & 2407.46 & 151929713 \\
\hline HLCS & 2422.99 & 152889925 \\
\hline CRYAA & 2440.96 & 146540499 \\
\hline MX1 & 2456.15 & 144732426 \\
\hline ATP2C1 & 2465.51 & 141332311 \\
\hline rs29017591 & 2478.05 & 137963609 \\
\hline rs29024361 & 2490.13 & 136296259 \\
\hline rs29026838 & 2513.20 & 144906700 \\
\hline
\end{tabular}

Continued on next page 
N.B. Stafuzza et al.

13102

Table 2. Continued.

\begin{tabular}{lcc}
\hline Marker & Position on BBU1 (cR5000) & Position on Bos taurus genome (bp) \\
\hline rs29026456 & 2536.27 & 142785760 \\
rs29024604 & 2558.27 & 139456223 \\
NCK1 & 2573.81 & 134262058 \\
BP230018B20G10 & 2590.57 & 132497697 \\
FOXL2 & 2600.04 & 132085550 \\
rs29022779 & 2609.32 & 130916681 \\
RNF7 & 2621.86 & 129473169 \\
rs29027942 & 2630.88 & 128394472 \\
U2SURP & 2655.21 & 128037601 \\
CD96 & 2750.20 & 56951704 \\
rs43708463 & 2791.31 & 57086245 \\
COPB2 & 2832.62 & 131999543 \\
\hline
\end{tabular}

Table 3. Buffalo sequence scaffolds ordered according to the positions of the markers mapped on BBU1.

\begin{tabular}{|c|c|c|}
\hline Marker & Position on BBU1 $\left(\mathrm{cR}_{5000}\right)$ & Buffalo scaffold UMD_CASPUR_WB_2.0 \\
\hline $\mathrm{SH} 2 \mathrm{D} 4 \mathrm{~A}$ & 42.52 & NW_005784720.1 \\
\hline THAP1 & 53.53 & NW_005785655.1 \\
\hline SLC20A2 & 64.54 & NW_005785655.1 \\
\hline ADRB3 & 140.00 & NW_005784334.1 \\
\hline BRF2 & 167.14 & NW_005784334.1 \\
\hline NRG1 & 241.32 & NW_005785682.1 \\
\hline DCTN6 & 255.61 & NW_005784975.1 \\
\hline WRN & 283.26 & NW_005785682.1 \\
\hline KIAA1456 & 309.04 & NW_005785635.1 \\
\hline ZDHHC2 & 342.32 & NW_005785878.1 \\
\hline CNOT7 & 355.23 & NW_005785878.1 \\
\hline MTNR1A & 374.29 & NW_005785680.1 \\
\hline TLR3 & 399.63 & NW_005784957.1 \\
\hline SLC25A4 & 416.35 & NW_005785619.1 \\
\hline CDKN2AIP & 475.07 & NW_005785619.1 \\
\hline DLGAP2 & 611.03 & NW_005784735.1 \\
\hline SLC5A3 & 669.05 & NW_005784098.1 \\
\hline IFNAR1 & 693.38 & NW_005784098.1 \\
\hline IFNGR2 & 724.07 & NW_005784098.1 \\
\hline KRTAP8 & 755.03 & NW_005784558.1 \\
\hline SOD1 & 808.70 & NW_005784534.1 \\
\hline TMPRSS15 & 1068.22 & NW_005784906.1 \\
\hline HSPA13 & 1102.32 & NW_005785817.1 \\
\hline POU1F1 & 1209.49 & NW_005785338.1 \\
\hline ST3GAL6 & 1257.68 & NW_005784065.1 \\
\hline FILIP1L & 1274.25 & NW_005784065.1 \\
\hline ALCAM & 1430.18 & NW_005784632.1 \\
\hline BBX & 1444.02 & NW_005784504.1 \\
\hline CASR & 1581.83 & NW_005785537.1 \\
\hline PDIR & 1648.84 & NW_005785826.1 \\
\hline KALRN & 1670.37 & NW_005785826.1 \\
\hline APOD & 1690.32 & NW_005784025.1 \\
\hline SST & 1762.02 & NW_005785332.1 \\
\hline CRYGS & 1770.67 & NW_005784864.1 \\
\hline SEC62 & 1908.87 & NW_005784729.1 \\
\hline IL12A & 2220.19 & NW_005785508.1 \\
\hline HLCS & 2422.99 & NW_005783537.1 \\
\hline CRYAA & 2440.96 & NW_005785117.1 \\
\hline $\mathrm{M} \times 1$ & 2456.15 & NW_005785734.1 \\
\hline ATP2C1 & 2465.51 & NW_005784738.1 \\
\hline NCK1 & 2573.81 & NW_005785398.1 \\
\hline FOXL2 & 2600.04 & NW_005785379.1 \\
\hline RNF7 & 2621.86 & NW_005785806.1 \\
\hline U2SURP & 2655.21 & NW_005785806.1 \\
\hline CD96 & 2750.20 & NW_005785563.1 \\
\hline COPB2 & 2832.62 & NW_005785379.1 \\
\hline
\end{tabular}




\section{DISCUSSION}

In the present study, we obtained a framework RH map of buffalo chromosome 1 based on cattle-derived markers. The BBU1 framework map presented, herein, provided a comparison of the gene order from buffalo chromosome 1 and bovine chromosomes 1 and 27 . The alignment of this RH map to the current bovine genome sequence assembly (Btau_4.6.1) indicated regions of possible rearrangements between the chromosomes of both the species. A set of linked markers on the telomeric region of BBU1q showed a rearrangement when compared to the sequence from bovine chromosome 1, revealing an inversion in the region flanked by the markers rs29021684 and COPB2 (Figure 1). In the previous RH maps constructed for this buffalo chromosome (Miziara et al., 2007; Amaral et al., 2008), an inversion in the same region was also observed when compared to the bovine chromosome 1 .

The RH map constructed in this study could provide a valuable tool to order scaffolds from the buffalo genome sequence, thereby contributing to the annotation of the buffalo genome, an important agricultural species whose genetic improvement has lagged behind other species because of limited genomic characterization previously.

\section{Conflicts of interest}

The authors declare no conflict of interest.

\section{ACKNOWLEDGMENTS}

Research funded by grants from FAPESP (São Paulo Research Foundation - Brazil; grant \#2011/11889-3) to MEJA and a post-doctoral fellowship to NBS (fellowship \#2011/02478-0).

\section{REFERENCES}

Agarwala R, Applegate DL, Maglott D, Schuler GD, et al. (2000). A fast and scalable radiation hybrid map construction and integration strategy. Genome Res. 10: 350-364.

Amaral ME, Owens KE, Elliott JS, Fickey C, et al. (2007). Construction of a river buffalo (Bubalus bubalis) whole-genome radiation hybrid panel and preliminary $\mathrm{RH}$ mapping of chromosomes 3 and 10. Anim. Genet. 38: 311-314.

Amaral ME, Grant JR, Riggs PK, Stafuzza NB, et al. (2008). A first generation whole genome RH map of the river buffalo with comparison to domestic cattle. BMC Genomics 9: 631.

Applegate D, Bixby R, Chvátal V and Cook W (1998). On the solution of traveling salesman problems. Doc. Math., extra volume ICM III 645-656.

Barendse W, Armitage SM, Kossarek LM, Shalom A, et al. (1994). A genetic linkage map of the bovine genome. Nat. Genet. 6: 227-235.

Everts-van der Wind A, Kata SR, Band MR, Rebeiz M, et al. (2004). A 1463 gene cattle-human comparative map with anchor points defined by human genome sequence coordinates. Genome Res. 14: 1424-1437.

Iannuzzi L, Di Meo GP, Perucatti A, Schibler L, et al. (2003). The river buffalo (Bubalus bubalis, $2 \mathrm{n}=50$ ) cytogenetic map: assignment of 64 loci by fluorescence in situ hybridization and R-banding. Cytogenet. Genome Res. 102: 65-75.

Jann OC, Aerts J, Jones M, Hastings N, et al. (2006). A second generation radiation hybrid map to aid the assembly of the bovine genome sequence. BMC Genomics 7: 283.

Ma RZ, van Eijk MJ, Beever JE, Guérin G, et al. (1998). Comparative analysis of 82 expressed sequence tags from a cattle ovary cDNA library. Mamm. Genome 9: 545-549.

Miziara MN, Goldammer T, Stafuzza NB, lanella P, et al. (2007). A radiation hybrid map of river buffalo (Bubalus bubalis) chromosome 1 (BBU1). Cytogenet. Genome Res. 119: 100-104.

Parma P, Erra-Pujada M, Feligini M, Greppi G, et al. (2004). Water buffalo (Bubalus bubalis): complete nucleotide mitochondrial genome sequence. DNA Seq. 15: 369-373. 
Schäffer AA, Rice ES, Cook W and Agarwala R (2007). rh_tsp_map 3.0: End-to-end radiation hybrid mapping with improved speed and quality control. Bioinformatics 23: 1156-1158.

Stafuzza NB, Abbassi H, Grant JR, Rodrigues-Filho EA, et al. (2009). Comparative RH maps of the river buffalo and bovine $Y$ chromosomes. Cytogenet. Genome Res. 126: 132-138.

Stafuzza NB, Abbey CA, Gill CA, Womack JE, et al. (2012). Construction and preliminary characterization of a river buffalo bacterial artificial chromosome library. Genet. Mol. Res. 11: 3013-3019.

Stafuzza NB, Borges MM and Amaral-Trusty MEJ (2014a). Comparative analysis of the river buffalo somatostatin gene. Genet. Mol. Res. 13: 10017-10024

Stafuzza NB, Borges MM and Amaral-Trusty ME (2014b). Sequence characterization and comparative analysis of the gastrotropin gene in buffalo (Bubalus bubalis). Genet. Mol. Res. 13: 10934-10942.

Stafuzza NB, Borges MM and Amaral-Trusty MEJ (2014c). Genetic analysis of superoxide dismutase 1 gene in murrah river buffalo. J. Anim. Plant Sci. 24: 1869-1875.

Untergasser A, Nijveen H, Rao X, Bisseling T, et al. (2007). Primer3Plus, an enhanced web interface to Primer3. Nucleic Acids Res. 35: W71-W74.

Voorrips RE (2002). MapChart: software for the graphical presentation of linkage maps and QTLs. J. Hered. 93: 77-78. 